\title{
VHE photon propagation and determination of the cosmological constants
}

\section{Oscar Blanch Bigas*}

IFAE, Campus UAB, E-08193 Bellaterra, Spain

E-mail: blanch@ifae.es

Photons produced in astrophysical objects need to travel through the galactic and intergalactic media before reaching the observer. Those having Very High Energy (VHE; usually defined as energies above $100 \mathrm{GeV}$ ) will interact with the background light, magnetic fields and the gravitational vacuum as well as may convert to Axion Like Particles. Hence, the flux of photons reaching the observer will not only have information about the phenomena happening in the astrophysical source but also on the medium they have traveled through. For extragalactic objects, these photons will undergo those interactions over cosmological distances, which will provide relevant information on both Cosmology and Fundamental Particles Physics. Among other results on these fields, the observations of VHE gamma rays have led to lower limits on the Quantum Gravity scale of $E_{Q G}>1.5 \cdot 10^{19} \mathrm{GeV}$ and $E_{Q G}>3.0 \cdot 10^{10} \mathrm{GeV}$ for leading order proportional to $O\left(E / E_{Q G}\right)$ and $\left(O\left(E / E_{Q G}\right)^{2}\right.$, respectively. The VHE gamma-ray data have also been shown to provide indirect measurements of cosmological constants. It includes a systematic dominated measurement of the Hubble constant that led to $H_{0}=71.8_{-5.6}^{+4.6}(\text { stat })_{-13.8}^{+7.2}($ sys $) \mathrm{km} \mathrm{s}^{-1} \mathrm{Mpc}^{-1}$. The better sensitivity and reduced systematic uncertainties of CTA should improve these results in the near future.

Science with the New Generation of High Energy Gamma-ray experiments, 10th Workshop - Scineghe2014 04-06 June 2014

Lisbon - Portugal

\footnotetext{
* Speaker.
} 


\section{Introduction}

The field of Very High Energy (VHE; > $100 \mathrm{GeV}$ ) gamma-ray astronomy has undergone a breakthrough in the last decade, mainly led by telescopes that use the Imaging Air Cherenkov Technique (IACT): HESS [1], MAGIC [2] and VERITAS [3]. From the detection of a handful of sources by their predecessors (HEGRA [4] and Whipple [5]), the number of detected astrophysical sources has grown to more than hundred sources thanks to the current generation of IACT telescopes. Water Cherenkov Observatories like MILAGRO [6], which stopped data taking in 2008, and HAWC [7], which is just starting to take physics data, also contribute to improve the knowledge on how the sky behaves in the VHE regime. The Fermi Gamma-ray Space Telescope [8] launched in 2008 covered the energy range just below the IACTs being of great help to better understand the high energy processes happening in the VHE gamma-ray astrophysical sources. All together they have led to a deeper knowledge on the astrophysical sources producing the most energetic particles in our universe. This knowledge will improve with the advent of CTA [9] in the near future.

The VHE gamma rays produced in the astrophysical sources will undergo interactions with the intergalactic and galactic media while traveling from the astrophysical sources to the observer. Therefore, the flux of photons reaching the Earth do not only contain information on the phenomena happening in the astrophysical sources but also an imprint of the interactions that they had suffered while traveling through the universe. Those coming from extragalactic sources will travel cosmological distances. The long distances travelled together with the VHE of the photons allow to extract information on both Cosmology and Fundamental Particle Physics from the imprint of the interactions in the photon flux.

The flux of VHE gamma rays that travel through the universe is attenuated by the absorption of gamma rays in the diffuse Extragalactic Background Light (EBL) through the Quantum Electrodynamics (QED) interaction $\gamma_{V H E} \gamma_{E B L} \rightarrow f^{+} f^{-}$. The cross section for this reaction decreases as the inverse of the square of the final state fermion mass and, hence, other final states than $e^{+} e^{-}$ are negligible. This leads the observed flux to be attenuated in respect to the original flux by a factor $\exp [-\tau(E, z)]$ where $E$ is the energy of the VHE photon at redshift $0, z$ the redshift of the astrophysical source where the photon was produced and $\tau$ is the optical depth of the gamma rays that can be computed as:

$$
\tau(E, z)=\int_{0}^{z} d z^{\prime} c \cdot \frac{d t}{d z^{\prime}} \int_{0}^{2 \pi} \sin \theta d \theta \int_{\frac{2 m^{2} c^{4}}{E\left(1+z^{\prime}\right)^{2}(1-\cos \theta)}}^{\infty} d \varepsilon n\left(\varepsilon, z^{\prime}\right) \sigma\left[2 E \varepsilon\left(1+z^{\prime}\right)^{2}(1-\cos \theta)\right]
$$

where $\frac{d t}{d z}$ is the lookback time; $\theta$ is the gamma-gamma scattering angle; $m$ is the fermion mass; $\varepsilon$ is the energy of the EBL target photon; $n\left(\varepsilon, z^{\prime}\right)$ is the EBL spectral density at a given $z^{\prime}$ and $\sigma$ is the cross-section for $\gamma_{V H E} \gamma_{E B L} \rightarrow e^{+} e^{-}$.

The wavelength range of the EBL that is relevant for the interactions with the VHE gamma rays goes from $\sim 1 \mu \mathrm{m}$ to $\sim 100 \mu \mathrm{m}$. At these wavelength range the direct measurements of $n(\varepsilon)$ are challenging mainly because of the strong foreground emission (zodiacal light, airglow). Still the great progress in the observation of faint sources of EBL allows quite accurate modeling of $n(\varepsilon)$ including its dependence on the redshift [10]. The reconstruction of the energy spectrum with high resolution of the VHE flux from extragalactic sources allow to perform indirect measurements of $n(\varepsilon)$ through the imprint of the gamma-gamma interaction in the observed flux [11]. Alternatively, 
the attenuation of the flux can be used to measure the cosmological densities and the Hubble constant through the dependence of the lookback time on them [12]. All this indirect measurement related to cosmology need to make assumptions on the intrinsic spectra, look for a common trend from many sources or both.

Although photons are not deviated by the magnetic fields they encounter while traveling, the $e^{+} e^{-}$pairs produced by the interaction $\gamma_{V H E} \gamma_{E B L} \rightarrow f^{+} f^{-}$do. Those $e^{+} e^{-}$pairs will also loose energy via inverse Compton scattering and produce secondary gamma rays that would be detected with some time delay and slightly deviated from the original direction of the gamma rays produced in the astrophysical source. The detection of these secondary gamma rays or upper limits on their fluxes provide information about the magnetic fields the gamma rays have crossed in their path to the observer [13].

In addition to expected standard model interactions, like those mentioned above, other interactions which may alter the energy or state of the photon can in principle be probed. Gamma rays traversing cosmological distances should also notice the quantum fluctuations in the gravitational vacuum which unavoidably should happen in any quantum theory of gravitation. These fluctuations may occur on scale sizes as small as the Planck length $L_{P} \sim 10^{-33} \mathrm{~cm}$ or time-scales of the order of $t_{P} \sim 1 / E_{P}$, with $E_{P} \sim 10^{19} \mathrm{GeV}$. Hence, the gamma rays will experience a vacuum polarization correction, which should be very small $\left(O\left(E / E_{Q G}\right)\right.$ where $E$ is the energy of the gamma ray and $E_{Q G}$ is an effective scale for Quantum Gravity, which might be as large as $E_{P}$ ) but might become measurable after the gamma rays have traversed cosmological distances. In this Quantum Gravity scenario, the requirement of violation of the Lorentz-Invariance symmetry [14] emerges naturally, providing an energy dependent propagation speed for electromagnetic waves. Therefore, gammas of different energies being emitted simultaneously by a distant source should reach the observer at different times.

Axions were introduced in the framework of Quantum Chromodynamics as pseudoscalar particles that would solve the strong CP problem [15]. Afterwards they became candidates to be part of the cold dark matter $[16,17,18]$. On the context of understanding the constituents of dark matter, similar but more generic particles were introduced: axion-like particles (ALPs) [19]. ALPs could couple to photons via a two-photon vertex, just as the axion does. Although, the mass and coupling constant for the ALPs are independent. The VHE photons are also expected to get mixed with possible ALPs. This coupling leads to the conversion of VHE gamma rays to ALPs and vice-versa while traveling through the universe from the source to the observer. This conversion will lead to alterations in the spectra observed [20].

This paper will center on the imprints of the interaction that the VHE photons undergo while traveling through the universe that provide information on Cosmology and Fundamental Particle Physics, which are not discussed elsewhere in these proceedings. These are namely the tests of Lorentz Invariance and the measurements of cosmological constants.

\section{Test of Lorentz Invariance}

When developing any model for Quantum Gravity, it appears naturally the necessity to modify some of the most basic continuous symmetries of spacetime, such as Lorentz Invariance [21, 22, 23]. The violation of the Lorentz Invariance symmetry (LIV) modifies the dispersion relation 
giving the propagation speed for gamma rays in a theory dependent manner. However, since in all plausible approaches the actual effects are expected to be small, they can be studied from a phenomenological point of view using an expansion in terms of the gamma energy divided by the effective Quantum Gravity scale $\left(E_{Q G}\right)$. Therefore, the actual gamma dispersion relation for a massless particle can be expressed in leading order as:

$$
E^{2}-c^{2} \vec{p}^{2} \simeq \xi\left(\frac{E}{E_{Q G}}\right)^{\alpha}
$$

where $E$ and $\vec{p}$ denotes the energy and the momentum of gammas, and $\xi$ and $\alpha$ are the LIV free parameters.

In this scenario, gamma rays traveling in vacuum can be seen as traversing a material medium [24]. Hence, the propagation speed of a gamma ray that reaches the Earth with energy $E_{\gamma}$ when it was at a redshift $z$ is:

$$
v=\frac{d E}{d p}=c\left[1+\xi \frac{1+\alpha}{2}\left(\frac{E}{E_{Q G}}\right)^{\alpha}\right]
$$

Therefore, the time needed for gamma rays to travel from the astrophysical source to the observer will depend on their energy and the time propagation difference when they reach the Earth can be expressed as:

$$
\Delta t=t_{E_{\gamma}}-t_{E_{\gamma}^{\prime}}=\int_{0}^{z}\left(\frac{c}{v_{E_{\gamma}}}-\frac{c}{v_{E_{\gamma}^{\prime}}}\right) \frac{d t}{d z} d z \simeq-\xi \frac{1+\alpha}{2} \frac{E_{\gamma}^{\alpha}-E_{\gamma}^{\prime \alpha}}{E_{Q G}^{\alpha}} \int_{0}^{z}(1+z)^{\alpha} \frac{d t}{d z} d z
$$

To be able to extract some information on LIV from the detected VHE photons, one needs to to know the difference between the times of their production in the astrophysical sources. Then one could translate their different arrival time to the observer to a different propagation time. This can be done by using fast variable phenomena as flaring Active Galactic Nuclei (AGN), Gamma Ray Bursts (GRBs) or pulsars. Still there, the photons belonging to the fast transient could have an intrinsic time-energy relationship at their productions site. Therefore, any claim about LIV based on energy dependent time lag observed on the photons detected by gamma observatories would need to be confirmed by different transients (different type, redshift or energy range).

The power of VHE gamma rays to measure or constrain the energy of the Quantum Gravity scale was already used by the Whipple collaboration with the pioneering IACT telescope Whipple. The data from a TeV gamma-ray flare from the AGN Mrk 421 observed on 15 May 1996 was used to place bounds on the possible energy dependence of the speed of light in terms of an effective scale for quantum gravitational effects. That data led to a lower limit at $95 \%$ confidence level (CL) of $4 \cdot 10^{16} \mathrm{GeV}$ [25] for $\alpha=1$. The limits were improved by the current generation of IACTs with a flare from Mrk 501 detected by MAGIC in 2005 [26] and from PKS 2155-304 detected by HESS in 2006 [27], leading to the current best limits with VHE photons: $E_{Q G}>2.1 \cdot 10^{18} \mathrm{GeV}$ and $E_{Q G}>6.4 \cdot 10^{10} \mathrm{GeV}$ for $\alpha=1$ and $\alpha=2$ respectively at $95 \% \mathrm{CL}$. The improvement came from both a new analysis method [28] and the better sensitivity of the instruments. Since then, other fast flares from AGNs detected by IACTs have not improved the limits and most likely improvement from VHE gamma-rays will only come with the future Cherenkov Telescope Array (CTA) [9], although HAWC with its capability to detect GRB may also provide stronger constraints. Currently, the best constraints from gamma rays come from observations of High Energy (HE; HE $>0.1 \mathrm{GeV}$ ) gamma 
rays from the GRB 090510 with Fermi [29]: $E_{Q G}>1.5 \cdot 10^{19} \mathrm{GeV}$ and $E_{Q G}>3.0 \cdot 10^{10} \mathrm{GeV}$ for $\alpha=1$ and $\alpha=2$ respectively at $95 \% \mathrm{CL}$. All these lower limits on the effective Quantum Gravity scale obtained from VHE and HE gamma rays are shown in Figure 1.

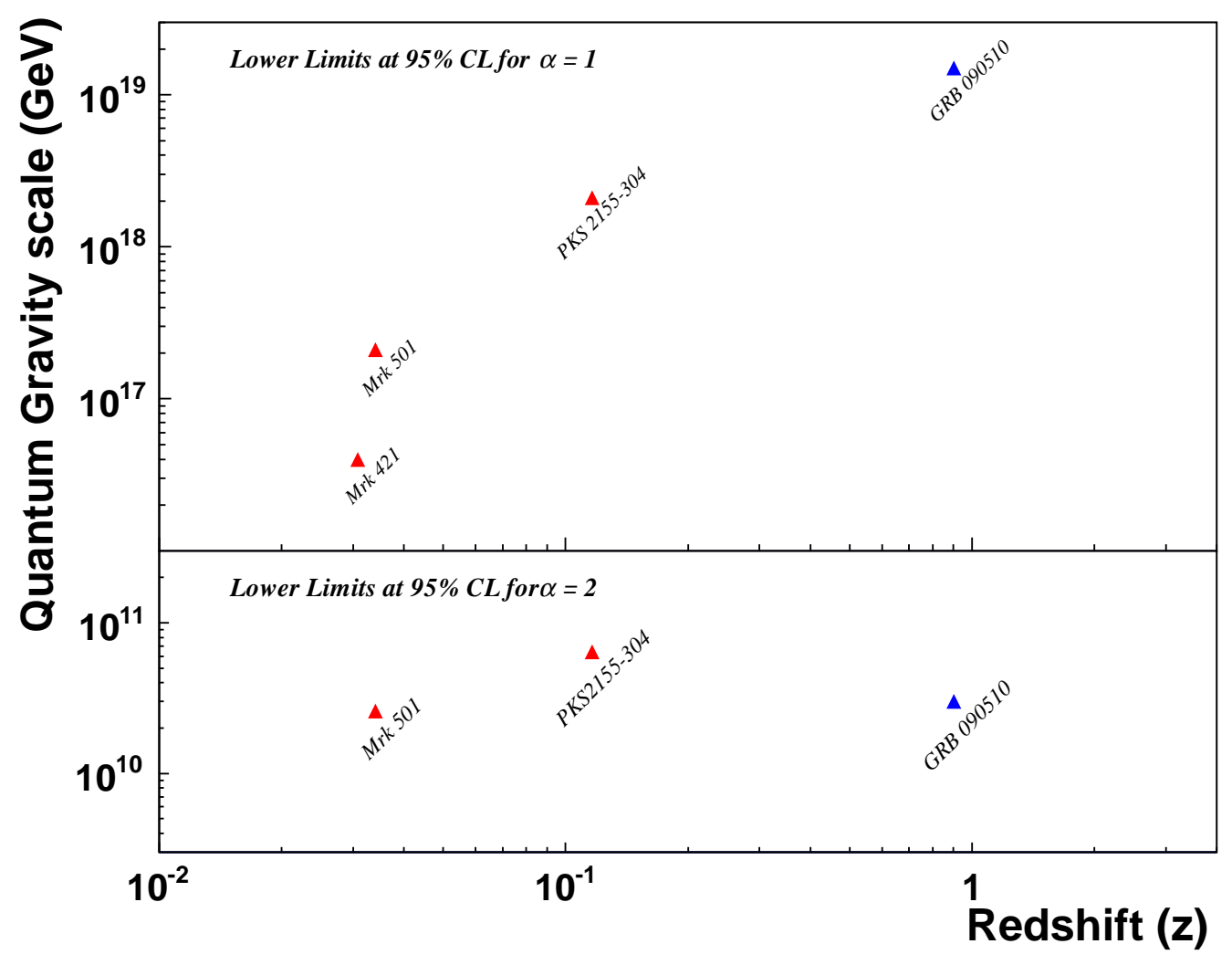

Figure 1: Lower limits for the effective Quantum Gravity scales derived from VHE and HE gamma-ray data. Result for both a linear $(\alpha=1)$ and quadratic $(\alpha=2)$ leading order on the gamma dispersion relation are shown. The lower limits coming from VHE gamma-ray data are derived from flaring AGNs: Mrk 421 [25], Mrk 501 [26] and PKS 2155-304 [27]. The lower limits involving also HE gamma-ray data are based on a Gamma Ray Burst: GRB 090510 [29].

Additionally, the modified gamma dispersion relation (Equation 2.1) would also lead to difference on the cross section for the QED interaction $\gamma_{V H E} \gamma_{E B L} \rightarrow f^{+} f^{-}$. Even assuming that the dynamics of the interaction keeps unaltered in a more general theory than QED, the threshold value for the gamma ray momentum to produce $e^{+} e^{-}$is modified [30], which leads to a modified energy threshold condition for the EBL photon in Equation 1.1 [31]:

$$
\varepsilon_{t h r}=\frac{2 m^{2} c^{4}}{E(1-\cos \theta)\left(1+z^{\prime}\right)^{2}}+\xi \frac{2}{\left.(1-\cos \theta) 1+z^{\prime}\right)} \frac{\left[E\left(1+z^{\prime}\right)\right]^{1+\alpha}}{4 E_{Q G}^{\alpha}}\left(\frac{1}{2^{\alpha}}-1\right)
$$

The modified threshold energy leads the universe to have a higher transperency at $\mathrm{TeV}$ energies [31] producing a signature in the observed spectra for extragalactic sources that could be in the reach for CTA [32] . 


\section{Determination of Cosmological Constants}

In the framework of the Standard Model of particle interactions, high energy gamma rays traversing cosmological distances are expected to be absorbed by their interaction with the diffuse EBL, producing $e^{+} e^{-}$pairs. The $\gamma_{H E} \gamma_{E B L} \rightarrow e^{+} e^{-}$cross section is strongly peaked at $E_{C M} \sim$ $1.8 \times\left(2 m_{e} c^{2}\right)$ and therefore, there is a specific range in the EBL energy which is probed by each gamma ray energy [33]. The imprint on the measured VHE spectra of this absorption, which can be estimated following Equation 1.1, has extensively been used to put constraints and try to measure the EBL [11].

Alternatively, the imprint of the absorption of a gamma-ray flux while traveling from the source to the observer could be used to measure Cosmological Constants [12, 34]. The power to get information on cosmological constants comes from the dependence of the lookback time on the cosmological densities $\left(\Omega_{M}, \Omega_{K}\right.$ and $\left.\Omega_{\Lambda}\right)$ and the Hubble constant $\left(H_{0}\right)$ :

$$
\frac{d t}{d z}=\frac{1 /(1+z)}{H_{0}\left[\Omega_{M}(1+z)^{3}+\Omega_{K}(1+z)^{2}+\Omega_{\Lambda}\right]^{1 / 2}}
$$

The precision one could get for the Cosmological Constants is not better than the ones one can obtain with other methods like SNe, CMB fluctuations or baryon acoustic oscillations $[35,36]$ even with the future generation of IACT. Still, the method provides an independent observable with different systematic uncertainties. The predictions for the Optical Depth and the Gamma Ray Horizon $(\mathrm{GRH} ; \tau(E, z)=1)$ normalized to their values at $z=0.01$ are shown in Figure 2. For comparison, the $z$ variation of the Luminosity-Distance, used for the determination of the cosmological parameters using $\mathrm{SNe}$ observations and of the Geodesical-Distance, giving the gamma ray path length, are shown. One can see that the Optical Depth has a quite different behavior depending on the gamma ray energy explored. To give a feeling of the actual average $z$ dependence of the Optical Depth, the prediction for an hypothetical EBL spectrum that does not depend on the wavelength of the EBL photons is also shown.

The observations of VHE photons with the current IACTs together with the complementary input provided by Fermi at HE, has lead to the first experimental evidence of the GRH [37], which is consistent with the predictions of the state-of-the-art EBL models [10]. This detection has been used to extract a measurement for the Hubble constant: $H_{0}=71.8_{-5.6}^{+4.6}(\text { stat })_{-13.8}^{+7.2}($ sys $) \mathrm{km} \mathrm{s}^{-1}$ $\mathrm{Mpc}^{-1}$ [38], where the systematics only include those coming from the method used to compute the GRH and do not include the systematics of the VHE observations themselves. Those systematics together with the knowledge of the EBL are two of the main limitations of the method to perform precision measurements. The effect of the former will be reduced with the future observatory of IACTs: CTA, for which a huge effort to reduce systematics is being done. The need to disentangle the imprint in the observed spectra due to the EBL absorption and those features that are intrinsic to the spectra, as for instance a possible cut-off, is also a limitation. Additionally, to get information on the cosmological densities one needs energy spectra reaching $\sim 100 \mathrm{GeV}$ with good statistics from sources at redshift $z \sim 1$, since the sensitivity to them is maximal there. CTA is expected to provide hundreds of AGNs detected with reasonable energy spectra from $\sim 10 \mathrm{GeV}$ to energies above $100 \mathrm{GeV}$, some of them at redshift larger than $z=1$ [39]. Hence, it will help to disentangle 


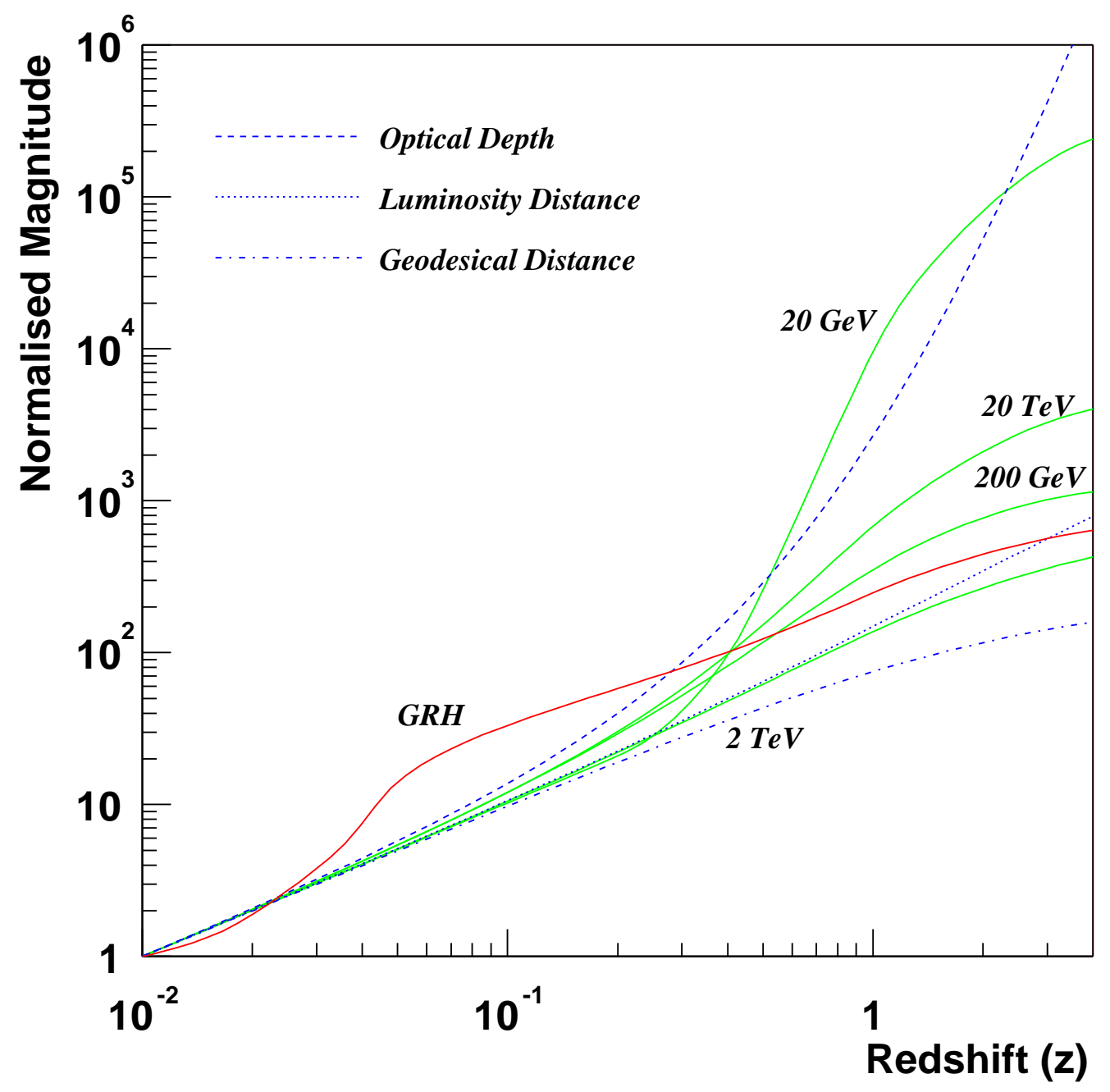

Figure 2: Redshift dependence of different observables. The predictions are normalized to their value at $z=0.01$. The solid lines correspond to the Optical Depth prediction for gamma rays of different energies ( $20 \mathrm{GeV}$ to $20 \mathrm{TeV}$ ). The dashed blue shows the hypothetical dependence with redshift of the Optical Depth if the EBL density would not depend on the wavelength. The GRH curve gives the $z$ dependence of the inverse of the GRH energy. (Figure from [34])

possible intrinsic features and will enable the possibility to measure the cosmological densities [40].

\section{References}

[1] https://www.mpi-hd.mpg.de/hfm/HESS/

[2] https://magic.mpp.mpg.de/

[3] http://veritas.sao.arizona.edu/

[4] A. Konopelko et al., Astropart.Phys.10 (1999) 275. 
[5] J. Lidea et al, Astropart.Phys. 28 (2007) 182.

[6] Milagro Collaboration, AIP Conf.Proc. 558 (2001) 549

[7] S. Westerhoff for the HAWC Collaboration, Adv.Space Res. 53 (2014) 1492

[8] http://fermi.gsfc.nasa.gov/

[9] Acharya et al, Astropart.Phys. 43 (2013).

[10] See contributions from A. Franceschini and A. Dominguez in these proceedings.

[11] See contribution from D. Mazin in these proceedings.

[12] O. Blanch and M. Martínez, Astropart.Phys.23 (2005) 588.

[13] See contribution from A. Neronov in these proceedings.

[14] G. Amelino-Camelia et al, Nature 393 (1998) 763.

[15] R.D. Peccei and H.R. Quinn, Phys. Rev. Lett. 38 (1977) 1440.

[16] J. Preskill et al, Phys. Lett. 120B (1983) 127.

[17] L.F. Abbott and P. Sikivie, Phys. Lett. 120B (1983) 133.

[18] M. Diee and W. Fischler, Phys. Lett. 120B (1983) 137.

[19] K.A. Olive et al. (Particle Data Group), Chin. Phys. C38 (2014) 09001.

[20] See contributions from A. Lindner, D. Horns and F. Tavecchio in these proceedings.

[21] S. Coleman and S.L. Glashow, Phys Rev. D59 (1999) 116008.

[22] G. Amelino-Camelia et al, Nature 393 (1998) 763.

[23] G. Amelino-Camelia and T. Piran, Phys Lett. B497 (2001) 265.

[24] J.I. Latorre, P. Pascual and R. Tarrach, Nucl. Phys. B437, (1995) 60.

[25] Biller et al, Phys.Rev.Lett. 83 (1999) 2108.

[26] J.Albert et al, Phys.Lett.B 668 (2008) 253.

[27] HESS, Astropart.Phys. 34 (2011) 738.

[28] M. Martínez and M. Errando, Astropart.Phys. 31 (2009) 226.

[29] Abdo et al, Nature 462 (2009) 331.

[30] G. Amelino-Camelia and T. Piran, Phys. Rev. D64 (2001) 036005.

[31] Blanch et al, Astropart.Phys. 19 (2003) 245.

[32] M. Fairbairn et al, JCAP 1406 (2014) 005.

[33] Vassiliev, V., Astropart. Phys. 12 (2000) 217.

[34] O. Blanch and M. Martínez, Astropart.Phys. 23 (2005) 597.

[35] P.A.R. Ade et al, Astron.Astrophys. (2014)

[36] Amanullah et al, Ap.J 716 (2010)

[37] A. Dominguez et al, Astrophys.J 770 (2013) 77.

[38] A. Dominguez and F. Prada, Astrophys.J. 771 (2013) L34

[39] H. Sol et al, Astropart.Phys. 43 (2013) 215.

[40] D. Mazin et al, Astropart.Phys. 43 (2013) 241. 